\title{
The Calculations of Dispersion Coefficients Inside Two-dimensional Randomly Packed Beds of Circular Particles
}

\author{
Amir Jourak $^{1}$, Vilnis Frishfelds ${ }^{1}$, T. Staffan Lundström ${ }^{1}$, Inga Herrmann ${ }^{2}$, Annelie Hedström² \\ ${ }^{1}$ Division of Fluid Mechanics, Luleå University of Technology, Luleå, email: Amir.Jourak@Ltu.se \\ ${ }^{2}$ Department of Civil, Environmental and Natural Resources Engineering, Luleå University of Technology.
}

This study investigates longitudinal and transverse dispersion in two-dimensional (2D) randomly packed beds of thousands of circular particles in a laminar flow regime over a range of superficial liquid velocities. This 2D discrete system of particles is divided into cells using modified Voronoi diagrams in which each cell contains one particle. The relationship between the average vorticity and the alteration of the stream function is obtained by using data from particular configurations of the three nearest particles. The stream function distribution in the system is then obtained by using the principle of energy dissipation rate minimization.

Then a finite packed-bed in which a concentration-type condition is set at the inlet boundary is mimicked by adapting boundary conditions to the rectangular $2 \mathrm{D}$ domain. With this system transient longitudinal dispersion numerical experiments were performed, and concentration profiles were fitted simultaneously with analytical solutions to estimate the values of the longitudinal dispersion coefficient $\left(D_{L}\right)$. Steady-state transverse simulations were performed in the 2D planar domain by adapting fixed concentrations on the side walls of the $2 \mathrm{D}$ porous medium in which the flow is directed upwards, and the values of the transverse dispersion coefficient $\left(D_{T}\right)$ were calculated from the approximate analytical solutions to that setup.

The $D_{L}$ was found to be scale-dependent, wherein far from the inlet, it increased in a fairly linear manner to the end of the bed. The advection-dispersion equation with a constant $D_{L}$ for the 2D packed-bed model was not able to represent the long tailings of the breakthrough curves. The estimated $D_{L}$ and $D_{T}$ were compared to some three-dimensional (3D) experimental data. At very low superficial liquid velocities, both longitudinal and transverse dispersions were observed to be governed by molecular diffusion. The values of $D_{L}$ that were obtained by the present $2 \mathrm{D}$ model agreed with $3 \mathrm{D}$ experimental data. At very high superficial liquid velocities, the 2D numerical experiments overpredicted $D_{T}$ a lot as compared to the $3 \mathrm{D}$ experiments. This is most likely a result of the $2 \mathrm{D}$ approach. 